\title{
MATHEMATICAL MODEL OF THE DEVELOPMENT OF MANUFACTURING DEFECTS IN THE SURFACE LAYER OF SUBSTRATES OF MOEMS' FUNCTIONAL COMPONENTS
}

\author{
Igor NEVLIUDOV ${ }^{1}$ (B) , Murad OMAROV ${ }^{2}$ (B) , Olena CHALA ${ }^{1}$ \\ ${ }^{1}$ Department of Computer-Integrated Technologies, Automation and Mechatronics, Kharkiv National University of Radio \\ Electronics, Kharkiv, Ukraine \\ ${ }^{2}$ Faculty of Automatics and Computerized Technologies, Kharkiv National University of Radio Electronics, Kharkiv, \\ Ukraine
}

\begin{abstract}
A mathematical model of the development of manufacturing defects, with the prediction of the random component of the model in the substrates of functional components of MOEMS, which are made of semiconductors, in particular, silicon, are developed in the article.

The main manufacturing defects that arise in the surface layer of the substrates of the MOEMS functional components taking into account the technological processes of their production and dynamic processes were used when developing the model. The developed mathematical model takes into account the occurrence of a random component of the model with its predictive ability.

The possibility of such control is the basis for the development of the scientific direction of technology and equipment for the production of semiconductors, materials and electronic devices - defect engineering, based on the management and forecasting of defect formation processes.
\end{abstract}

Keywords: Mathematical model, Defect, MOEMS, Functional components

\section{INTRODUCTION}

MOEMS components rank second in terms of production in the world among MEMS components. The main functional component of MOEMS is the optical component Micro Mirror, it is usually a silicon substrate coated with a metallized layer or without it, which provides a high reflectivity of the light beam when it is redirected in optical fibers $[1,2]$. One of the characteristics of such a functional MOEMS component, which are of primary importance, is defectiveness.

Suppose a functional component has been manufactured and its parameters comply with the norms. But, it should be borne in mind that the practice of operating the functional components of MOEMS shows that the development of defects arising during its manufacture (operation) is one of the main causes of failures and incorrect operation of the device and manifests itself in time, that is, in dynamics. It is also necessary to take into account an unknown component, which is the technical operation conditions of the device as a whole or unaccounted for or unforeseen parameters.

Currently, there are a large number of methods and tools to identify manufacturing defects, but the capabilities of these methods are limited, given the time component and the unknown component [3-9].

Control and test operations that are part of the structure of modern technological processes cannot give a complete guarantee of the absence of defects in the production of this type of components and their behavior in time, taking into account the operating conditions [6]. 
In this regard, the problem arises of predicting and monitoring the technical state of the functional components of MOEMS, which is estimated by monitoring the parameters that change over time. The resulting information reflects the development of manufacturing defects, which in turn do not always worsen the parameters of microsystems [10-15].

Improvement of the information display method makes it possible to observe changes in the technical state of the MOEMS functional components.

It is the adequate models of the development process of manufacturing defects of the functional components of MOEMS that will make it possible to make decisions on the guaranteed prediction of incorrect operation and parametric failures in time [12, 16-19].

Thus, the development of mathematical models for predicting the development of defects in functional components of MOEMS, modeling and monitoring changes in their physical and technological parameters, taking into account the temporal (dynamic) and unknown component, is an urgent task in microsystem technology.

\section{INFLUENCE OF CONSTRUCTIVE AND TECHNOLOGICAL FACTORS OF THE PRODUCTION TECHNOLOGY OF FUNCTIONAL SUBSTRATES OF MOEMS COMPONENTS ON THE KINETICS OF DEFECT FORMATION}

Since the functional component of the MOEMS switch is Micro Mirror, having considered its structure [20-22], we conclude that defects in such functional components at the production stage are based on defects of three groups:

1) $\Omega_{S}$ functional component substrate defects;

2) defectiveness of the $\Omega_{c}$ metalized layer;

3) defectiveness of diffusion of technological alignment when obtaining such structures $\Omega_{d}$.

Taking this into account, it is possible to represent a mathematical model of a set of manufacturing defects of functional components of MOEMS in the form (1):

$$
\Omega_{m d}=\Omega_{s}+\Omega_{c}+\Omega_{d} .
$$

Most of the manufacturing defects arise precisely because of the defectiveness and (or) impurities in the starting materials of the substrates or sublayers of substrates of the functional components of MOEMS.

Therefore, it was decided to consider the substrate defects of the MOEMS functional component as the main and initial source of defects and defects in the MOEMS component as a whole.

The problem arises in the fact that at the stage of production of starting materials, it seems unlikely that it is possible to track the defectiveness of structures and the dependence of physical and technological parameters, which directly affect the quality and compliance of the output characteristics, a special limitation is imposed by the factor of the kinetics of degradation processes in materials during and operating conditions.

But nevertheless, the main reason for the limited resource characteristics of the functional components of MOEMS are manufacturing defects that develop during the operation of the MEMS.

There are a large number of control methods for detecting defects [5-13]. To prevent the defects, the work is being carried out to provide diagnostic support for technological equipment and technological processes [17-20]. A system for monitoring manufacturing defects exists, but despite the large number 
of methods and means for detecting defects, some of them remain undetected. At the same time, the availability of these tools and their high information content provide a variety of information about the parameters of the functional components of MOEMS, its use makes it possible to improve monitoring and, on this basis, adjust the manufacturing technology.

Analysis of the data suggests that the mechanism of the occurrence of manufacturing defects in the functional components of MOEMS and the tasks associated with their development is based on the transformations of the micro- and macrostructure of the initial materials and chemical transformations that occur in the production and operation of functional components of MOEMS [1-5]. In this case, the scheme of transformation of sub microdefects and microdefects into macrodefects is realized.

1. The diffusion of layers and sublayers of functional MOEMS components can be expressed using Fick's second law: for one-dimensional diffusion (2) or diffusion through a film (3):

$$
\begin{gathered}
\frac{d V}{d t}=D \frac{d^{2} V}{d x^{2}}, \\
\frac{d V}{d t}=D \frac{\Delta V}{y} .
\end{gathered}
$$

where $D-$ is a diffusion coefficient;

$V$ - concentration of diffusing substance (components);

$y$ - film thickness.

It should be noted that the thermal oxidation of silicon is of no small importance. Let's consider the option that a mathematical model is built on the use of a silicon substrate as a functional component of MOEMS.

Thermal oxidation of silicon occurs due to the diffusion of oxidizing components $\left(\mathrm{O}_{2}, \mathrm{H}_{2} \mathrm{O}\right)$ through the oxide to the interface $\mathrm{Si}-\mathrm{SiO}_{2}$, where the oxidation reaction takes place [22-27].

The kinetics of the oxidation process is described by the model developed by Dill and Gurov [27]. Fig.1 shows a silicon substrate covered with an oxide layer and which is in contact with the gas phase [27-28].

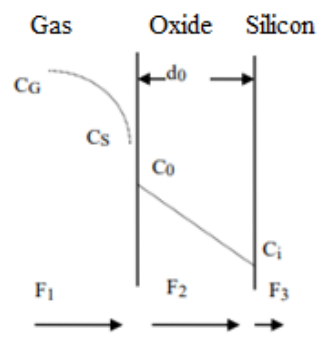

Figure 1. Model of thermal oxidation of silicon

Considering the above, we can conclude that oxidation is an unequal process, the driving force of which is the deviation of concentration from equilibrium. The flow of these oxidizer particles through the oxide for any point $V$ of the oxide layer is described by the law (1). Oxidation during the production of substrates in such structures of MOEMS functional components is a particular case of diffusion. 
2. Chemical corrosion of layers and sublayers of functional MOEMS components, more precisely, the corrosion rate, can be represented in the form (4), and in the presence of protective films, the reaction rate (5) is:

$$
\begin{gathered}
\frac{d V}{d t}=V_{0} e^{-\frac{E}{R T},} \\
\frac{d V}{d t}=\frac{k_{d} k_{p}}{k_{d}+k_{p} h_{0}} V_{0} .
\end{gathered}
$$

where $E$ is an activation energy of molecules participating in the reaction;

$k_{p}$ - chemical reaction rate constant;

$V_{0}$ - concentration of a reactant (for example, oxygen) on the outer surface at the interface with the

gas phase;

$h_{0}$ - coating thickness;

$k_{d}-$ corrosion diffusion coefficient.

3. The evaporation rate of the substrate material or sublayers of MOEMS functional components can be expressed as (6):

$$
V^{\prime}=\frac{k_{p}}{\sqrt{2 \pi R}} / p \sqrt{\frac{M}{T}} .
$$

where $M$ - molecular weight of the vaporized substance;

$p$ - pressure;

$R$ - gas constant;

$T$ - absolute temperature.

Given that all the above-described transformations of the micro-and macrostructure of the source materials and chemical transformations take place over time, it is necessary to take into account the speed of these reactions.

Diffusion and chemical reactions can serve as the basis for determining the rate and, consequently, describing the kinetics of the processes under consideration.

Statistical physics makes it possible to explain the kinetics of thermodynamic parameters of a medium based on the behavior of a set of particles that this medium consists of.

The microscopic state of a collection of particles is completely determined by the canonical variables (X). From the macroscopic point of view, the state of matter is determined by a very limited number of parameters sufficient for the macroscopic characteristics of the medium. Setting these parameters, measured in a macroscopic experiment, determines the macroscopic state of the medium. Macroscopic parameters, including the volume of the reacted substance, are functions of the canonical variables:

$$
V_{k}(X) \text {, and } k=1,2, \ldots, n \text {, where, } n<<\text {. }
$$

Based on macroscopic measurements, only statistical judgments can be made about the values of microscopic variables $X$. 
Thus, the macroscopically specified system is depicted by setting the probability density of the variables $\omega(X, t)$.

This phase probability density is sometimes also called the phase probability distribution, or simply the phase distribution.

Knowing $\omega(X, t)$, you can calculate the statistical average value $V(X)$ according to the formula (7):

$$
V=\int_{(X)} V(X) \omega(X, t) d X,
$$

as well as the standard deviation (8):

$$
\Delta V=\sqrt{\overline{(V-\bar{V})^{2}}} .
$$

manifested as a fluctuation of the observed area of the PW of the reacted substance.

The correspondence between the behavior of an aggregate of substrate particles and the region of the reacted substance can be illustrated in the figure (Fig. 2).

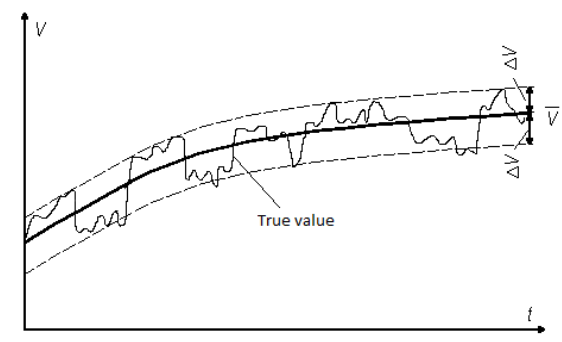

Figure 1. Influence of the behavior of the macroscopic parameter of a set of particles on the volume of the reacted substance

The average value $\bar{V}$ is shown here as a bold line. The true random value $V$ in this particular experience is represented by a thin irregular line.

Gibbs's apparatus of statistical mechanics makes it possible to derive general relations connecting mean values, variances, and generally quadratic correlations of generalized coordinates with their mean values in the presence of additional forces acting on these coordinates.

If the value $V(x)$ is a function of coordinates only, then it can be represented as a new generalized coordinate $q(X)$.

The value $a$ can be understood as an additional external force acting in the direction of the generalized coordinate $q$. The latter means that the Hamilton function of the system has the form (9):

$$
H(X, a)=H_{0}(X)+a q(X),
$$

Indeed, in this case, according to Hamilton's equations:

$$
\dot{p}=-\frac{\partial H}{\partial q}=-\frac{\partial H_{0}}{\partial q}-a=A_{0}-a .
$$

in other words, an additional force $a$ acts on the system in addition to the force $A_{0}=-\frac{\partial H_{0}}{\partial q}$. Substituting $H(X, a)$ from Gibbs' theory, for the generalized coordinates of system (9) in the expression [27-28] that is valid in the problem under consideration, we obtain: 


$$
\overline{(q-\bar{q})^{2}}=-k T \frac{\partial \bar{q}}{\partial a}
$$

The use of the main provisions of the Gibbs theory [27-28] makes it possible to calculate the average values and fluctuations of any physical quantities that are functions of coordinates, if the dependence of the average values of these quantities on external constant forces acting on them is known.

It is not possible to estimate these forces for specific objects. Here only one can draw a conclusion about the hierarchical nature of fluctuations with different orders of relaxation time for microscopic and macroscopic parameters.

Considering the process of transformation of a substance as a physicochemical reaction, evaluating the rate of the process through the change in the volume of the PW or NW per unit time, it can be assumed [54] that the rate of change in the concentration of that component (12):

$$
\frac{d C_{j}}{d t}=K \prod_{i} C_{i} r_{i}
$$

where $C_{i}$ is the concentration of the $i$-th component involved in the reaction;

$r_{i}$ - indicator, the total order of the reaction for the $i$-th substance, it can be equal to zero, be an integer or fractional number.

For an adequate description of the processes, we will restrict ourselves to orders equal to $0,1,2$. The rate constant $K$ is one of the main parameters of the process, essentially determining the relaxation time and therefore is called the relaxation constant.

For our mapping, the following hypotheses are accepted:

- two-component composition of the medium (two-component substrate);

- dependence of the rate of the process on the volume $W_{0}-V$ of $\mathrm{NW}$, where $W_{0}$ - is the initial volume of the unreacted substance and $V$ - is the volume of the unreacted substance;

- the rule of proportionality between the amount of a substance and its concentration when distributed over the volume of the medium. Then the considered volume of the reacted substance and the unreacted substance can be perceived as the concentration of the components participating in the reactions, which can be systematized into a list of technological defects in each separate technological process, indicating the cause of the defect and subsequent mechanisms of deviation of the parameter values from the specified ones or failure of the substrate materials of the functional components;

- the assumption that during the course of reactions the thermodynamic coefficients can also depend on the state of the medium, reflecting the possibility of using the Onsager equation [27-28] for nonlinear processes.

Then the equation of the process combining the thermodynamic and physical-technological approaches can be represented in the form (13):

$$
\frac{d V}{d t}=K\left(W_{0}-V\right)^{r}=f(V),
$$

where $r$ is the order of the reaction, it can be zero or one for a linear model and two for a nonlinear model. 
The solution of this equation can be used to find the deterministic part of the time dependence of the volume of the elementary region of the reacting substance.

Analysis of the data, the list of technological defects in each individual technological process, indicating the cause of the defect and subsequent mechanisms of deviation of the parameter values from the specified ones or refusal in the substrate materials of the functional components of MOEMS (carried out by the authors), confirms the possibility of using equation (13) for the identified mechanisms of development processes of production defects, so for:

- diffusion at $D$ proportional to the concentration of the unreacted substance $W_{0}-V, r=2$ at constant $D$ one can take $r=1$ or $r=0$;

- various types of corrosion, taking into account the dependence of the activation energy and constant reactions on the concentration of the unreacted substance, $r=2$, for a linear description $r=0$ or $r=1$;

- evaporation of material $r=0$.

For the zero-order reaction, the following differential equation is used (14):

After integration, the reaction equation is obtained (15):

$$
V=K t .
$$

If we take the time of observation of the process as the origin of time, the change $V(\delta t)=\left|\delta x^{i} \delta x^{j}\right|$ observed in the observation interval $\delta t$ can be used to calculate the relaxation constant $K$, that is, the time period for which the amplitude value of the disturbance in the unbalanced physical system decreases by a factor of $e$.

Hence the relaxation constant is (16):

$$
K=\frac{V(\delta t)}{\delta t} .
$$

To display the kinetics of the process under consideration for the first order of the reaction, the following differential equation is used (17):

$$
\frac{d V}{d t}=K\left(W_{0}-V\right)
$$

After integration, we get (18):

$$
V=W_{0}\left(1-e^{-K t}\right) .
$$

For this case, the relaxation constant is (19):

$$
K=\frac{\ln \left(W_{0}\right)-\ln \left(W_{0}-V(\delta t)\right)}{\delta t} .
$$

For a reaction order of two, the following differential equation is used (20):

$$
\frac{d V}{d t}=K\left(W_{0}-V\right)^{2} .
$$

After integration, the reaction equation looks like this (21): 


$$
V=W_{0}+\frac{W_{0}}{1+K t W_{0}} .
$$

For this case, the relaxation constant is (22):

$$
K=\frac{\frac{1}{W_{0}-V(\delta t)}-\frac{1}{W_{0}}}{\delta t} .
$$

Since the result of the process is $W_{0}=V$, we can assume that $W_{0}$ is determined by the system of equalities (23):

$$
\left.\begin{array}{l}
W_{0}=a^{i} a^{j} \\
a^{i}=x^{i}\left(t_{n}\right)-x_{\text {min }}^{i}, \text { если } \delta x^{i}<0 \\
a^{i}=x^{i}{ }_{\max -x^{i}}\left(t_{n}\right), \text {,если } \delta x^{i} \geq 0
\end{array}\right\} .
$$

The lack of information about the reaction order leads to the problem of choosing it based on observations. The selection criterion can be the maximum value of the errors $\Delta V$ obtained as the difference between the observed and calculated from the table 1 sized area.

Table 1 Differential equations and their solutions for reactions of various orders

\begin{tabular}{ccc}
\hline $\begin{array}{l}\text { Reaction } \\
\text { order }\end{array}$ & $\begin{array}{c}\text { Differential } \\
\text { equations of } \\
\text { reactions }\end{array}$ & $\begin{array}{c}\text { Reaction } \\
\text { equation after } \\
\text { integration }\end{array}$ \\
\hline 0 & $\frac{d V}{d t}=K$ & $V=K t$ \\
1 & $\frac{d V}{d t}=K\left(W_{0}-V\right)$ & $V=W_{0}\left(1-e^{-K t}\right)$ \\
2 & $\frac{d V}{d t}=K\left(W_{0}-V\right)^{2}$ & $V=W_{0}+\frac{W_{0}}{1+K t W_{0}}$ \\
\hline
\end{tabular}

Based on the interval estimation of the mathematical expectation $M_{n}(\Delta V)$ of errors at the moment $t_{n}$ and that $\max _{n} \Delta V$ is located on the border of the obtained interval, for the same selection conditions for all models characterized by the reliability of estimates (24):

$$
\max _{n} \Delta V=M_{n}(\Delta V)+\frac{\sqrt{D_{n}(\Delta V)}}{\sqrt{n}} .
$$

where $D_{n}(\Delta V)$ is the variance of errors at the moment $t_{n}$.

The solution of the problem of choosing a mathematical model, therefore, provides for calculating the maximum value of errors for each order of reactions and the choice of such an order that provides the minimum value of errors.

Thus, statistical physics makes it possible to explain the kinetics of the thermodynamic parameters of the medium on the basis of the behavior of a set of particles of the substrates that make up the submarine, and the macroscopic state of the particles and the change reflect the behavior of the reacted substance of the functional substrate of the MOEMS component. 
It can be concluded that the fluctuations have a hierarchical nature, which have different orders of relaxation time for microscopic and macroscopic parameters.

As a result, an evolutionary differential equation is proposed that describes the reactions occurring in the observed medium of the defective upper layer of the functional substrate of the MOEMS component; it is used to find the deterministic part of the time dependence of the volume of the elementary region of the reacted substance of the functional substrate of the MOEMS component.

\section{PREDICTION OF THE RANDOM COMPONENT OF THE DEVELOPMENT MODEL OF MANUFACTURING DEFECTS OF THE SURFACE LAYER OF THE FUNCTIONAL SUBSTRATE OF THE MOEMS COMPONENT}

To display fluctuations, taking into account the impossibility of fully using the results of statistical physics due to the lack of the necessary quantitative information about the Hamiltonian of a set of interacting particles, it seems possible to use the methods of statistical analysis of the results of observations.

Since the purpose of such an analysis is prediction, one can use the theory of extrapolation of random processes, in particular, the methods of optimal filtering.

A specific feature of the solution to the problem associated with the use of optimal filtering to isolate and predict the random component of the model under consideration is the nonlinearity of evolutionary equations [29-30].

A linearization of the problem is proposed based on the concept of a reference trajectory and the expansion of a nonlinear function $f(V)$ in a Taylor series.

As a reference trajectory, sufficiently close to the true one, solutions $V_{O n}(t)$ of differential equations for various orders of reactions are used, then a random additive correction $Z$ to the deterministic $V_{\text {on }}$ is subject to evaluation and prediction, which is a solution of differential equations for the true trajectory $V(t)$ characterizing the process of development of manufacturing defects of functional substrates of MOEMS components (25):

$$
V(t)=V_{\text {On }}(t)+Z(t) .
$$

Amendment estimation methods assume knowledge of the law of its distribution. Random processes of changing the parameters of functional substrates of MOEMS components are usually considered to be normally distributed.

The validity of the hypothesis of the normal distribution of parameters is confirmed by many years of research experience in the theory of accuracy and experimental data on technological deviations of the parameters, for which the greatest factual material has been accumulated.

The technological spread of parameters determines the distribution of the random process of the drift of parameters at the initial moment of time (in the section $t=0$ ).

For monotonic processes, the type of the initial distribution in the first approximation is preserved for any other time sections [31-32]. 
The use of Gaussian random processes is to some extent justified by the fact that the normal distribution law contains the maximum entropy in comparison with any continuous distribution with the same variance. Therefore, replacing some distribution with a normal one will not overestimate the estimates.

The final form of the evolution equation is (26):

$$
\frac{d V_{\text {On }}}{d t}+\frac{d z}{d t}=f\left(V_{\text {on }}\right)+\frac{d f(V)}{d V} \Delta V .
$$

where $f\left(V_{\text {on }}\right)$ - the left side of the differential equations corresponds to the system of equations,

$$
\frac{d V_{\text {on }}}{d t}=f\left(V_{\text {on }}\right) \text {, }
$$

assuming $z=\Delta V$

$$
\frac{d z}{d t}=\frac{d f(V)}{d V} z,
$$

Considering the solution of the problem of predicting a random component based on the Kalman extrapolation algorithm, which provides for the use of models of the evolution of a random component and observation at discrete times specified by the number $k$.

The initial conditions are determined when $k=0$, when $k=1$ observation is performed, prediction is performed for $k=2$. These models are determined by the equations $(29-30)$ :

$$
\begin{gathered}
z(k+1)=\frac{d f(V)}{d V} z(k)+w(k), \\
g(k)=z(k)+v(k),
\end{gathered}
$$

where $w(t)$ is normal white noise with zero average value $\operatorname{cov}\{\omega(t), \omega(\tau)\}=d_{w} \delta(t-\tau)$,

$\delta(t-\tau)-$ Dirac delta function,

$v(t)$ - process due to observation errors.

The nature of the process $w(t)$ is due to fluctuations in the parameters of nonequilibrium media, corresponding to the main provisions of statistical physics, which makes it possible to substantiate the behavior of a set of micro particles that determine the thermodynamic parameters of the media of which they are composed. As a consequence of this behavior, you can see thermal noise, Brownian motion and other similar phenomena.

In the problem under consideration, the noise characterizes the probabilistic blurring of the boundaries of the reacted substance, caused by fluctuations of its size, which are random.

Here we can also see an analogy with a real environment, which consists of a set of micro particles distributed over energies, for example, the canonical Gibbs distribution for an isothermal system, the Maxwell-Boltzmann distribution, as a consequence of this distribution, for an ideal gas, which makes it possible to give a "mechanical" interpretation of thermodynamic parametersm.

It can be assumed that the initial values of the random component $z(0)$ and the initial variance $d_{w}$ are determined by the mean values $z(t)$ and variance obtained at the previous moments of observations.

Extrapolation equations have the form (31): 


$$
z(k+1 / k)=\frac{d f(V)}{d V} z(k / k-1)+K(k+1, k)[g(k)-z(k / k-1)],
$$

with a gain of $K(k+1, k)$, defined from the equation (32):

$$
K(k+1, k)=\frac{d f(V)}{d V} D(k / k-1)\left[D(k / k-1)+D_{V}\right]^{-1},
$$

where $D_{v}$ is the variance due to observation errors.

The variance equation has the form (33):

$$
\begin{aligned}
& D(k+1 / k)=\left(\frac{d f(V)}{d V}\right)^{2} D(k / k-1)+d_{w}- \\
& -\left(\frac{d f(V)}{d V} D(k / k-1)\right)^{2}\left[D(k / k-1)+D_{V}\right]^{-1}
\end{aligned}
$$

Obviously, the obtained expressions based on the results of observations $g(k)$ at the corresponding $k=1$ time point allow us to estimate the value $z(\delta t p)=z(k+1 / k)$ and $D(\delta t p)=D(k+1 / k)$ after the forecast time interval $\delta t_{p}$ corresponding $k+1=2$, i.e. make a forecast of the development of the process and evaluate the forecast error.

Then it can be argued that knowledge of the mathematical expectation $z\left(\delta t_{p}\right)$ and variance $D\left(\delta t_{p}\right)$ makes it possible to make a parametric estimate of the normal distribution law of a random variable. Based on the obtained distribution density function $\omega(z)$, it is possible to estimate the probability $P$ of a prediction error, or vice versa, the error interval $\Delta_{z}$ for a given probability of finding an error in this interval, using this probability as a guarantee of decisions about the technical state of the functional substrates of MOEMS components, using the expression (34):

$$
P\left(-\Delta_{z}<z<+\Delta_{z}\right)=\int_{-\Delta_{z}}^{+\Delta_{z}} \omega(z) d z,
$$

Real estimates corresponding to the beginning of the process can be made only after a set of statistical data (roughly $n>4$ ) regarding the deviation of the real trajectory from that calculated on the basis of the selected model, the corresponding mathematical expectations are naturally selected as estimates [3337].

The random component $z$ is observed in the form of forecast errors, then it can be assumed that at the initial moment of estimation $t_{n}(k=0)(35-36)$ :

$$
\begin{gathered}
z(0 / 0)=M_{n}(\Delta V), \\
d_{w}=D(0 / 0)=D_{n}(\Delta V),
\end{gathered}
$$




\section{CONCLUSIONS}

Thus, the article presents a mathematical model of the development of manufacturing defects in the surface layer of substrates of MOEMS functional components.

A mathematical model based on data on the main manufacturing defects that arise in the surface layer of substrates of MOEMS functional components, in the technological processes of their production, taking into account dynamic processes.

The developed mathematical model takes into account the emergence of a random component of the model with its predictive ability.

The possibility of such prediction and control is the basis for the development of a scientific direction in technology and equipment for the production of semiconductors, materials and electronic devices defect engineering, based on the management and prediction of defect formation processes.

The results obtained should be taken into account in the development of technological processes for the production of electronic devices, in particular microsystems based on MOEMS components.

\section{REFERENCES}

[1] Guyon I, Elisseeff A. An introduction to variable and feature selection. J Mach Learn Res 2003; 3: $1157-1182$.

[2] Izadpanahi S, Ozcınar C, Anbarjafari G, Demirel H. Resolution enhancement of video sequences by using discrete wavelet transform and illumination compensation. Turk J Elec Eng \& Comp Sci 2012; 20: 1268-1276.

[3] Sabanskis A, Virbulis J. Modelling of thermal field and point defect dynamics during silicon single crystal growth using CZ technique //Journal of Crystal Growth. - 2019. - T. 519. - C. 7-13.

[4] M. Wu and W. Wong, "Numerical Approach to Predict Silicon Wafer Grinding Warpage, 2018 20th International Conference on Electronic Materials and Packaging (EMAP), Clear Water Bay, Hong Kong, 2018, pp. 1-3, doi: 10.1109/EMAP.2018.8660847.

[5] Filipenko O, Chala O. i Videshyn M. 2017. Технологічні дефекти виробництва кремнієвих підкладок для функціональних відбиваючих поверхонь моемс-перемикачів. Системи управління, навігащіï та зв'язку. Збірник наукових пращъь. 2, 42 (Груд 2017), 61-63.

[6] Невлюдов И. Ш. Проектные решения повышения надежности кремниевых интегральных преобразователей механических величин / И. Ш. Невлюдов, М. А. Омаров, К. Ю. Харенко // Радиотехника : Всеукр. межвед. науч.-техн. сб. - Х. : ХНУРЭ, 2006. - Вып. 147. - С. 119 122.

[7] Филипенко О. І. Технологічні фактори виробництва, що впливають на якість покриттів дзеркальних поверхонь МОЕМС-перемикачів / О. І. Филипенко, О. О. Чала, М. І. Відешин // Наукові нотатки. - 2017. - Вип. 57. - С. 178-183

[8] Talla JA. Electronic properties of silicon carbide nanotube with Stone Wales defects under uniaxial pressure: a computational study //Computational Condensed Matter. - 2019. - T. 19. - C. 
[9] Justo JF. et al. Interatomic potential for silicon defects and disordered phases //Physical review B. -1998. - T. 58. - №. 5. - C. 2539.

[10] Филипенко О.І. Методи контролю структур топології поверхонь матеріалів виробів електронної техніки, МЕМС та МОЕМС / О.І. Филипенко, О.О. Чала, Ю.В. Бондаренко // Технология приборостроения. - 2018. - № 2. - С. 3-7.

[11] Abdelnaby H, Potirniche GP, Elshabini A, Barlow F, Groothuis SK and Parker,RS. Numerical Simulation of Heat Generation During the Back Grinding Process of Silicon Wafers, 2012 IEEE Workshop on Microelectronics and Electron Devices, Boise, ID, 2012, pp. 1-4, doi: 10.1109/WMED.2012.6202614.

[12] Wilson $\mathrm{M}$ et al., Importance of defect photoionization in silicon-rich SiNx dielectrics for high PID resistance, 2013 IEEE 39th Photovoltaic Specialists Conference (PVSC), Tampa, FL, 2013, pp. 0218-0222, doi: 10.1109/PVSC.2013.6744134.

[13] Margutti G et al., Silicon Defects Characterization for Low Temperature Ion Implantation and Spike Anneal Processes, 2014 20th International Conference on Ion Implantation Technology (IIT), Portland, OR, 2014, pp. 1-4, doi: 10.1109/IIT.2014.6940014.

[14] Seal,S, Budhraja V, Sopori B, Devayajanam S, Basnyat P and Varadan VV. Defect Mapping and Stress Mapping of Crystalline Silicon using Spectroscopic Ellipsometry, 2013 IEEE 39th Photovoltaic Specialists Conference (PVSC), Tampa, FL, 2013, pp. 0212-0217, doi: 10.1109/PVSC.2013.6744133.

[15] Zafirovska M, Juhl $\mathrm{K}$ and Trupke T. Comparison of Line Scan Luminescence Imaging Techniques for Defect Characterisation in Crystalline Silicon Solar Modules, 2018 IEEE 7th World Conference on Photovoltaic Energy Conversion (WCPEC) (A Joint Conference of 45th IEEE PVSC, 28th PVSEC \& 34th EU PVSEC), Waikoloa Village, HI, 2018, pp. 1364-1369, doi: 10.1109/PVSC.2018.8547434.

[16] L. J. Duan, H. H. Au, M. Kuan, P. S. Quek and K. S. Pey, A New Mechanism of Poly-silicon Crater Defect Induced from Al Tiny Particle Charging Effect during Water Rinse in Oxide Patterning Process, 2006 IEEE International Integrated Reliability Workshop Final Report, South Lake Tahoe, CA, 2006, pp. 198-200, doi: 10.1109/IRWS.2006.305245.

[17] Селиванова К. Г. Математическое моделирование электромиографического сигнала / К. Г. Селиванова, О. Г. Аврунин, А. А. Гелетка // Вестник Нац. техн. ун-та "ХПИ" : сб. науч. тр. Темат. вып. : Новые решения в современных технологиях. - Харьков : НТУ "ХПИ". - 2014. - № 36 (1079). - C. 31-39.

[18] Li Z, Jing X, Jiang F and Zhang W. Modeling and Simulation of Silicon Wafer Backside Grinding Process, 2014 15th International Conference on Electronic Packaging Technology, Chengdu, 2014, pp. 874-877, doi: 10.1109/ICEPT.2014.6922787.

[19] Sakhnenko NK, Stognii NP, Nerukh AG. Chipouline A., Pertsch T. Modeling of Transient Plasmon Dynamics In Metallic Cylinders // Proc. Int. Conference on Mathematical Method in Electromagnetic Theory (MMET 2012), Kharkiv, Ukraine. - 28 - 30 August 2012. - P. 35 - 38.

[20] Agarwal R, Samson, S, Kedia S and Bhansali S. Fabrication of Integrated Vertical Mirror Surfaces and Transparent Window for Packaging MEMS Devices, in Journal of 
Microelectromechanical Systems, vol. 16, no. 1, pp. 122-129, Feb. 2007, doi: 10.1109/JMEMS.2006.886010.

[21] Fujita T, Nagatani Y and Maenaka K. MEMS Mirror Controlling System with Holed-PSD, 2010 3rd International Conference on Emerging Trends in Engineering and Technology, Goa, 2010, pp. 446-449, doi: 10.1109/ICETET.2010.101.

[22] Filipenko O, Chala O and Sychova O. Some Issues of Dependencies of Loss from Technological Features of Optical Switches for Communication Systems, 2018 International Scientific-Practical Conference Problems of Infocommunications. Science and Technology (PIC S\&T), pp. 599-603, 2018.

[23] Prashant K, Dhavse R and Mishra V. Dependence of Oxidation Process on Various Oxidizing Conditions, 2014 2nd International Conference on Emerging Technology Trends in Electronics, Communication and Networking, Surat, 2014, pp. 1-4, doi: 10.1109/ET2ECN.2014.7044945.

[24] Hashim U et al., Comparison of Deal Grove Model Growth Rate with Dry Thermal Oxidation Process for Ultra-Thin Silicon Dioxide Film, 2015 2nd International Conference on Biomedical Engineering (ICoBE), Penang, 2015, pp. 1-4, doi: 10.1109/ICoBE.2015.7235905.

[25] Ma F et al., Modeling of Stress-Retarded Thermal Oxidation of Nonplanar Silicon Structures for Realization of Nanoscale Devices, in IEEE Electron Device Letters, vol. 31, no. 7, pp. 719-721, July 2010, doi: 10.1109/LED.2010.2047375.

[26] Agache V, Bigotte P, Legrand B, Senez V, Buchaillot L and Collard D, Modeling and Experimental Validation of Silicon Nanotip Oxidation: Towards A Nanoelectromechanical Filter Application, TRANSDUCERS '03. 12th International Conference on Solid-State Sensors, Actuators and Microsystems. Digest of Technical Papers (Cat. No.03TH8664), Boston, MA, USA, 2003, pp. 1287-1290 vol.2, doi: 10.1109/SENSOR.2003.1217008.

[27] Терлецкий Я. П. Статистическая физика: Учебное пособие. - Высшая школа, 1966. Технология С. Под ред. С. Зи; Пер. с англ., кн. 1. - 1986.

[28] Рудой Ю. Г., Суханов А. Д. Термодинамические флуктуации в подходах Гиббса и Эйнштейна //Успехи физических наук. - 2000. - Т. 170. - №. 12. - С. 1265-1296.

[29] Teh, WH. Boning DS and Welsch, RE. Multi-Strata Stealth Dicing Before Grinding for Singulation-Defects Elimination and Die Strength Enhancement: Experiment and Simulation, in IEEE Transactions on Semiconductor Manufacturing, vol. 28, no. 3, pp. 408-423, Aug. 2015, doi: 10.1109/TSM.2015.2438875.

[30] Smyntyna V, Kulinich O, Glauberman M, Chemeresuk G, Yatsunskiy I and Sviridova O. Influence of Initial Silicon Defects on Processes of the Dioxide Silicon Defect Formation, 2006 16th International Crimean Microwave and Telecommunication Technology, Sevastopol, Crimea, 2006, pp. 608-609, doi: 10.1109/CRMICO.2006.256126.

[31] Zhu Y, Coletti G and Hameiri Z Injection Dependent Lifetime Spectroscopy for Two-Level Defects in Silicon, 2019 IEEE 46th Photovoltaic Specialists Conference (PVSC), Chicago, IL, USA, 2019, pp. 0829-0832, doi: 10.1109/PVSC40753.2019.8981261.

[32] Doroshenko VO, Klimova NP, Stognii NP and Kostyn YD. Mathematical Modelling of Impulse Excitation of a Superwideband PEC Cone Antenna, 2019 IEEE 8th International Conference on 
Advanced Optoelectronics and Lasers (CAOL), Sozopol, Bulgaria, 2019, pp. 396-399, doi: 10.1109/CAOL46282.2019.9019509.

[33] Li D and Su J. Nondestructive Defect Detection and Localization of Defects in Annular Through Silicon Via(TSV), 2019 Cross Strait Quad-Regional Radio Science and Wireless Technology Conference (CSQRWC), Taiyuan, China, 2019, pp. 1-3, doi: 10.1109/CSQRWC.2019.8799141.

[34] Nevliudov İ, Omarov M, Botsman İ, Demska N, Nevliudova V, Starodubcev M. Research Of Factors Influencing The Process Of Formation Of Welded Microconnections In Electronic Modules. Eskişehir Technical University Journal of Science and Technology A - Applied Sciences and Engineering 2019, 20 () ,181-187. DOI: 10.18038/estubtda.651032.

[35] Zhang L, Dong Y and Wang J, Wind Speed Forecasting using a Two-Stage Forecasting System With an Error Correcting and Nonlinear Ensemble Strategy, in IEEE Access, vol. 7, pp. 176000176023, 2019, doi: 10.1109/ACCESS.2019.2957174.

[36] Wei L, Zhang Z, Ning Y and Lv J. Improved Markov Residual Error to Long-Medium Power Load Forecast Based on SVM Method, 2009 First International Workshop on Education Technology and Computer Science, Wuhan, Hubei, 2009, pp. 128-132, doi: 10.1109/ETCS.2009.38.

[37] Charan CR. Application of Generalized Neuron Model in Short Term Load Forecasting under error functions, 2010 Second International conference on Computing, Communication and Networking Technologies, Karur, 2010, pp. 1-4, doi: 10.1109/ICCCNT.2010.5591670. 